Biosight 2020; 01(02): 16-23

A. Ali et al.

\title{
Association of Some Demographic Factors with Undiagnosed Neuromuscular Insufficiencies; A Survey Report
}

\author{
Anwar Ali* and Mariam Asad
}

Department of Physiology, University of Karachi, Karachi, Pakistan.

Received: September 19, 2020 Accepted: October 20, 2020

\begin{abstract}
To investigate the factors that may contribute to develop Neuromuscular insufficiencies in the general population of Karachi like age origin, occupation, kind of work, daily water intake, daily working hours and exposure time to various screens etc. Questionnaire-based survey. 150 individuals ranging between 15-65 years were interviewed with the help of a self-designed questionnaire. The data was analyzed statistically by using Microsoft Excel. Neuromuscular indications and difficulties were positive in $79 \%$ of the interviewed individuals. A major number of subjects complained to have different neuromuscular insufficiencies including, body pain $(68 \%)$, joint pain (42\%), numbness (42\%) and muscle weakness $(63 \%)$. Some of the participants reported to suffer with the difficulties in walking (13\%), running (25\%), holding $(7 \%)$ and muscular strength (9\%). However, only $29 \%$ of these sufferers were formerly diagnosed with any neuromuscular disease with $18 \%$ had a family history of neuromuscular disorders. Remaining i.e., $71 \%$ are those who have not been diagnosed with any disorder and they were suffering with these symptoms. Among these un-diagnosed participants 53\% had consanguineous marriages among their parents. Those, who were reported to have any of the neuromuscular problem includes: $26 \%$ students, $31 \%$ office workers, $11 \%$ labours and $33 \%$ housewives. An important factor that has appeared to contribute mainly in the development of neuromuscular insufficiencies is the time for exposure to screens like TV, Computer, Cell phone etc. Among these undiagnosed participants who were suffering with neuromuscular problem(s), $93 \%$ are being exposed to these screens for more than 5 hours a day. Another most important factor was appeared to be the daily water intake as most $(88 \%)$ of those individuals' daily water intake was less than 10 glasses per day. Back pain was the major complaint observed through this data which seems to be related with prolonged inappropriate posture.
\end{abstract}

Keywords: neuromuscular disorder, muscular insufficiencies, body pain, screen hours

Corresponding Author: Anwar Ali Department of Physiology University of Karachi,

Karachi, Pakistan.Email: anali@uok.edu.pk

\section{Introduction:}

Neuromuscular disorders include heterogeneous conditions that may directly involve intrinsic muscle pathology or may occur indirectly due to any abnormality in the peripheral nervous system, neuromuscular junctions and motor cells in the spinal cord causing improper functioning of the muscles [1]. Neuromuscular disorders are responsible to impair the function of voluntary muscles and in most extreme cases can lead to complete paralysis [2].

The last $25^{\text {th }}$ years are said to be the vital era in the field of genetics because many genes were identified that are responsible for the neuromuscular disorder in the human being. Neuromuscular disorders may be inherited and run throughout the species as an autosomal dominant, autosomal recessive or $\mathrm{x}$-link trait. After the discovery of Duchene dystrophy gene in 1987, hundreds of gene were identified that were responsible for the cause of neuromuscular disorders. The age of onset of neuromuscular disorders occurs at any time from neonatal to old age.

Neuro-muscular pain is a multifaceted and discomfort. Chronic pain is a widespread problem among the general public. The rate of prevalence for chronic pain across different countries is about 7.3\%-12.9\%. Fibromyalgia is the most widespread chronic neuromuscular pain that has been reported by the general population. Fibromyalgia is defined as an idiopathic, cardinal musculoskeletal pain related to a different syndrome which has been characterized by muscular or joint aches with stiffness and tenderness [3].

In neuromuscular disorders, a patient may experience some complications in lower extremities includes difficulty in walking due to muscles weakness and loss of articular range of motion and upper extremities complications includes contracture that starts to develop in arms and hand during early stage with severe pain and discomfort [4]. Sometimes the difficulty in swallowing and breathing are also observed. Associated with such symptoms muscle wasting and muscle cramping particularly in proximal limb small muscles of hands are commonly observed in old aged people [5].

Consanguineous marriage is generally defined as a union between two people having blood relation, related as a second cousin or closer based on traditional, occupational and social groups known as "Biraderi" [6]. The word 


\section{Biosight 2020; 01(02): 16-23}

consanguinity is referred to as the sharing of genetic material. In Pakistan, the rate of consanguineous marriages is very high. Consanguineous marriages are mostly preferred in the region because of socio-cultural values.

In under-developing countries because of inadequate diversity of food, many individuals may suffer from various health problems. Malnutrition affects all groups of age but it is especially common in poor people who have an inadequate amount of food, clean water, health education and good sanitation.

Environmental pollution is one of the widespread problems nowadays, which affect the human population. Piosonous substances like pollutants in the air and water specifically causing the abnormalities of muscles in the growing children [7].

\section{Aims and Objectives of the Study:}

This study was designed to explore the possible relationship of neuromuscular insufficiencies with different demographic parameters so that a major factor could be identified that is causing the increasing number of neuromuscular abnormalities. Following parameters were set to be investigated for any relationship with neuromuscular abnormalities:

- Origin

- Occupation

- Kind of work and daily working hours

- Daily water intake

- Exercise routine

- Exposure to screens

\section{Materials and Method}

Study design and duration

This survey was carried out at the district city of Karachi, Pakistan between the month of October and January.

\section{Subjects}

We conducted a survey-based study involving 150 randomly selected individuals including 46 males and 104 females, between the age of 15 and 65 years.

\section{Inclusion criteria}

All the subjects were mentally and physically active and having a healthy lifestyle between the age of 15 and 65 , without being diagnosed for any neuromuscular disease.

\section{Exclusion criteria}

Drug addicted and those who have gone through any physical trauma in the last 5 years were excluded from the study

\section{Questionnaire Designing}

A questionnaire was designed containing questions regarding the presence of neuromuscular symptoms, frequency and intensity of the symptoms and the history of any neuromuscular disorder.

\section{Collection of Data}

Random subjects were selected to fill the questionnaire from different places of Karachi that include offices, schools, universities and homes. History details were recorded by asking the questions about socio-demographic variables including consanguineous marriages between their parents, origin, occupation, kind of work, daily working hours, daily water intake, daily computer and visual screen hours, balance diet, supplement intake, treatment, addiction, daily exercise, sports and any other disease that contribute to the neuromuscular disorder. Health examination has been recorded by asking them about some common indications such as body pain, joint's pain, back pain, numbness and general muscle weakness that were mostly observed in a patient of neuromuscular disorder with their frequencies (rare, sometimes, usual and most of the time) and intensity of these indications were identified by graded them as high, medium or low.

\section{Data analysis}

In the second part of the study we divided our subjects into three groups according to their ages, each group contain 50 individuals. Group 1 contain young aged subjects having ages between 15-25 years, group 2 contain middle-aged subjects having ages between 26-45 years and group 3 contain old aged subjects having ages between 46-65 years to collect data from all age group. All the data values were compared and presented in the form of an appropriate chart or graph.

\section{Results}




\section{Biosight 2020; 01(02): 16-23}

Indications of neuromuscular disorder

Figure 1 shows the data related to the various indications (signs and symptoms) of neuromuscular origin. According to our data, $68 \%$ of the interviewed persons found to have body pain, $42 \%$ subjects are reported to have joint pain, $49 \%$ for numbness and $63 \%$ reported for muscle weakness (Figure 1a). Frequencies of these indications have also been asked and shown in figure 1b. Finally, the intensity of these indications was also investigated and have been summarized in (figure 1c). Body pain was found to be low in $9 \%$, medium in $27 \%$ and high in $27 \%$ individuals. For joint pain, it was $6 \%$ low, $15 \%$ medium and $20 \%$ high. Back pain intensity includes $7 \%$ low, $18 \%$ medium and $27 \%$ high. Numbness intensity includes $7 \%$ low, $17 \%$ medium and $25 \%$ high. Muscles weakness intensity observed are $12 \%$ low, $33 \%$ medium and $19 \%$ high.

\section{INDICATIONS}

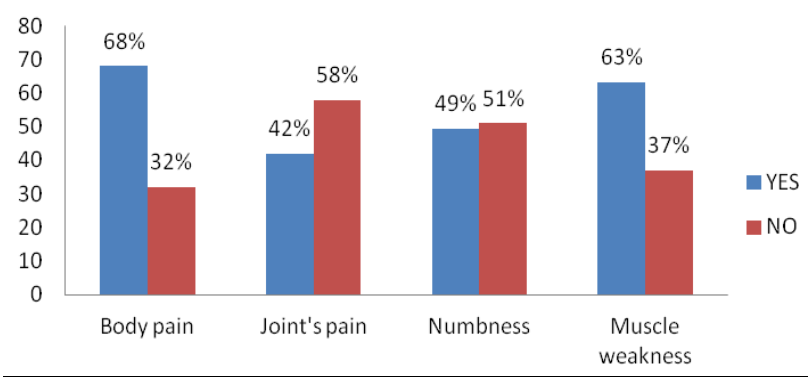

Figure 1a: Bar diagram showing various indications of neuromuscular disorders in percentages.

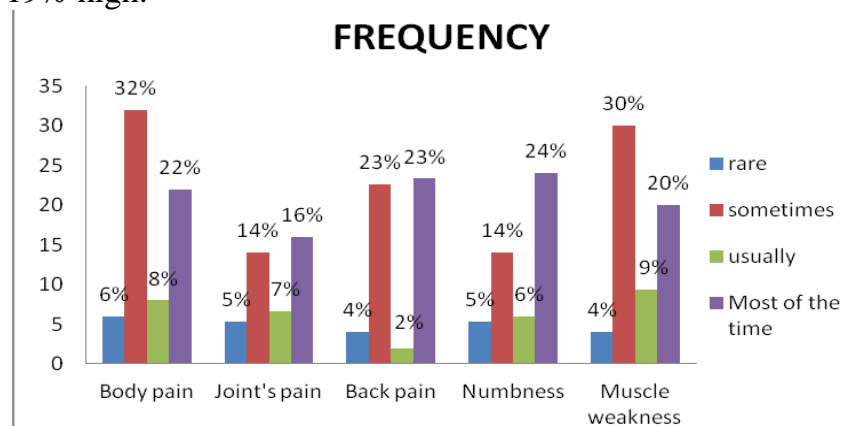

Figure 1b: Bar diagram showing the percentages frequencies for neuromuscular indications.

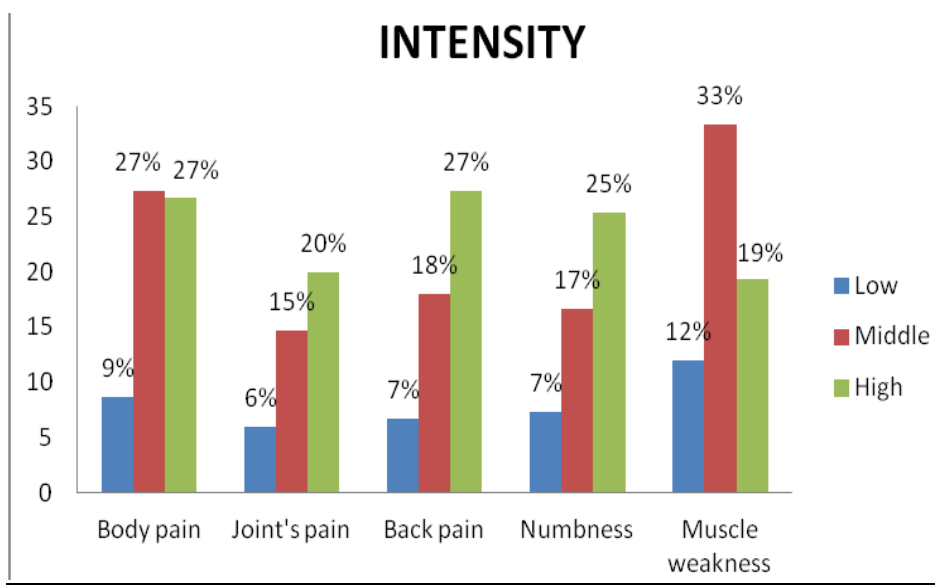

Figure 1c: Bar diagram showing the percentages for the intensity of neuromuscular indications.

Difficulties of muscular functions

We also asked if the individuals were facing any difficulty in walking, running holding and muscle strength. The obtained data has been shown as graph in (Figure 2). Difficulties identified in our data includes walking in $13 \%$, running in $25 \%$, holding $7 \%$ and strength difficulty in $9 \%$.

\section{DIFFICULTY}

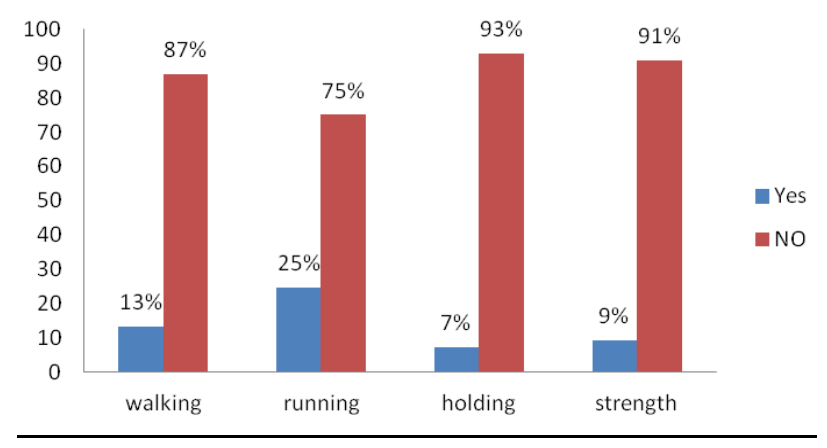

Figure 2: Bar diagram showing the percentages for difficulties in neuromuscular disorders.

Family history and consanguineous marriages 


\section{Biosight 2020; 01(02): 16-23}

According to our data (summarized in Figure 3) $18 \%$ of the individuals with positive neuromuscular insufficiencies had a family history of NMD. Further, among those participants who reported to be positive for any of the neuromuscular symptoms, 53\% found to have consanguineous marriage between their parents.

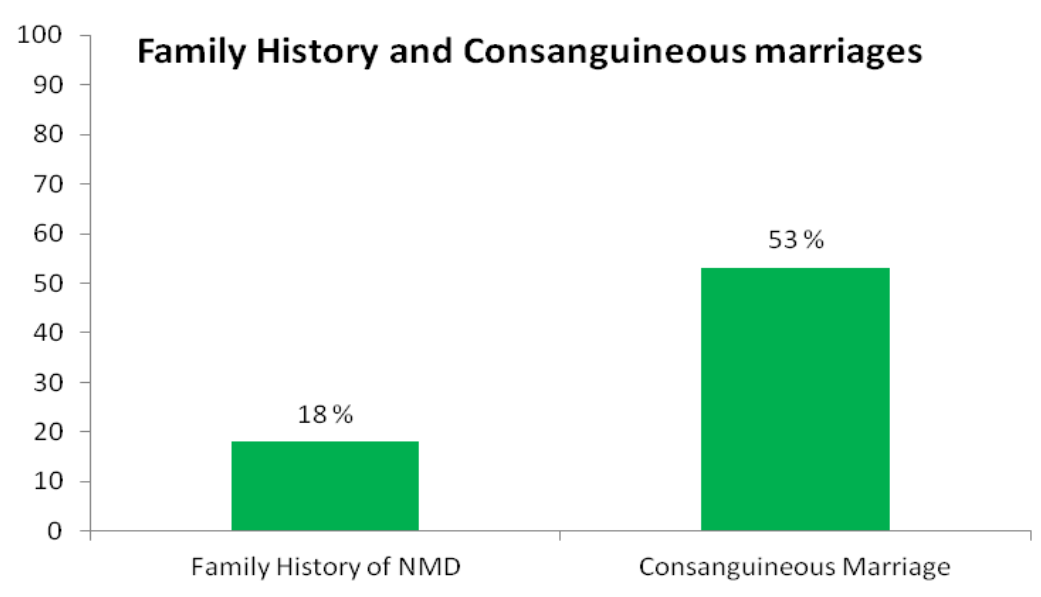

Figure 3: Bar diagram showing Family History and Consanguineous marriages

Origin

Individuals having positive symptoms belonged to different origins. Summary of the data is presented in Figure 4

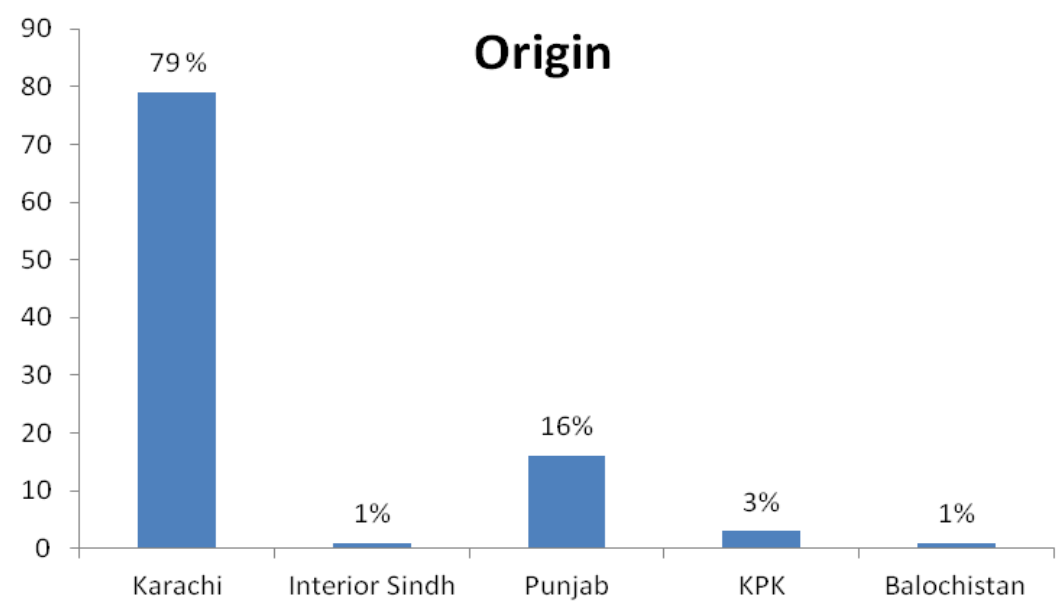

Figure 4: Bar diagram showing the origin of participants (with NMD indications).

Occupation

Figure 5 shows the percentages of the different occupation of those participants who reported any neuromuscular indications. Those included students (26\%), office workers (31\%), labours (11\%) and housewives (33\%).

\section{OCCUPATION}

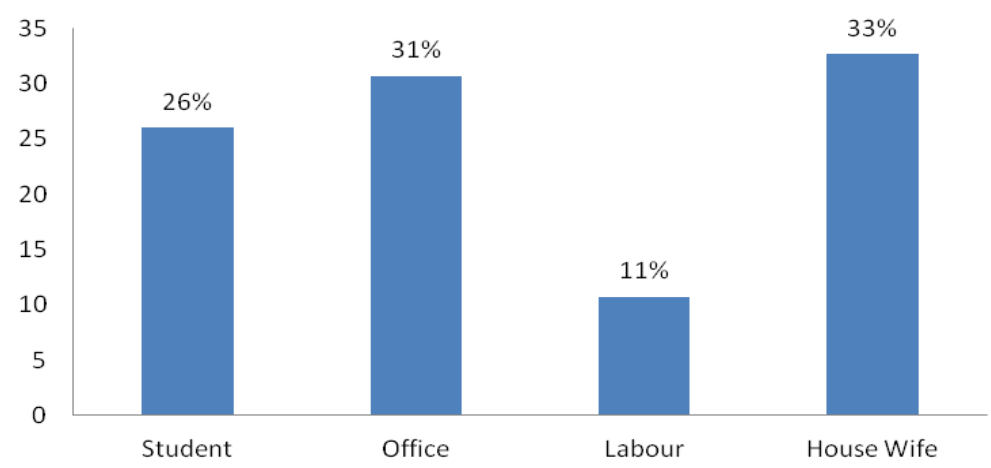

Figure 5: Bar diagram showing the percentages of occupation.

Kind of work 


\section{Biosight 2020; 01(02): 16-23}

Those individuals who were positive for neuromuscular indications were asked whether in their work place they remain standing or sitting or walking in most of the duration. Result has been shown in Figure $6.63 \%$ of those participants spend most of their time while sitting.

\section{KIND OF WORK}

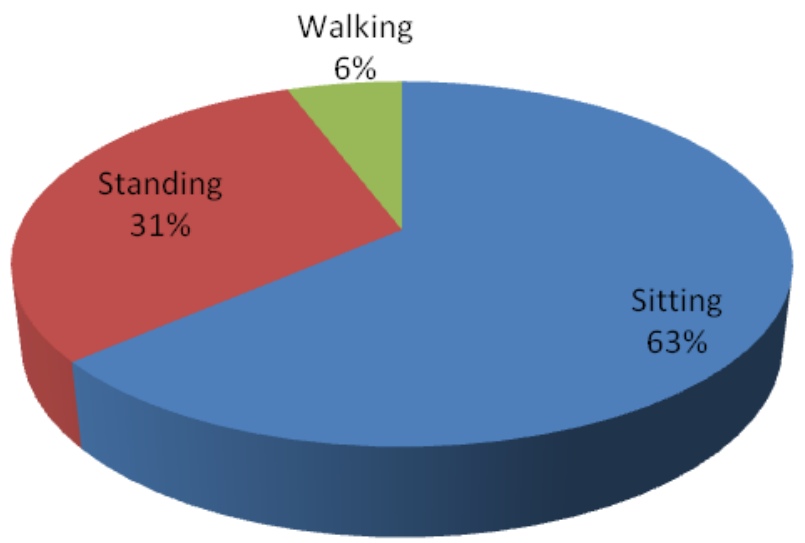

Figure 6: Pie chart showing the percentages of kind of work.

Daily water intake

According to our data, $41 \%$ of positive individual have water intake less than 5 glasses, $47 \%$ have $5-10$ glasses and $12 \%$ have greater than 10 glasses per day (Figure 7).

\section{DAILY WATER INTAKE}

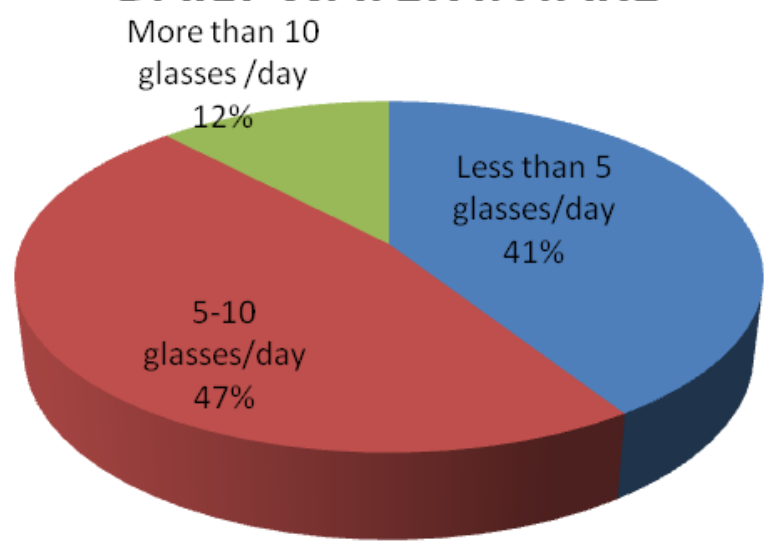

Figure 7: Pie chart showing the percentages of daily water intake.

Daily screen hours

According to our data, $93 \%$ of positive individuals spend 5 or more hours a day watching screens, including TV, LED, LCD, Laptop and Mobile phone.

\section{Exercise}

Another important relationship was between the positive signs of neuromuscular problems with the daily routine of exercise. $96 \%$ of the positive individuals do not follow a proper exercise routine.

\section{Discussion}

This was a pilot study, carried out among the small number of randomly selected individuals with different age groups and regardless of the gender. In this regard the results cannot be implemented on the population and further investigations with some more controlled parameters will be required. This study was a simple survey in order to determine the direct relationships of some demographic parameters with the neuromuscular insufficiencies. If we compare the obtained data, with a positive family history for NMDs, $24 \%$ of individuals were reported for indications of neuromuscular problems with $7 \%$ of them were reported to have difficulties in 


\section{Biosight 2020; 01(02): 16-23}

their daily life. If we consider age with the positive indications data showed positive individuals with young age $(23 \%)$, middle-age $(29 \%)$, and in old age is $(27 \%)$ whereas difficulties are also seen in young age $(5 \%)$, middleage $(10 \%)$ and old age $(17 \%)$. Individuals originated from Karachi were found to have more positive cases $(66 \%)$ as compared to others. As far as the kind of work is concerned, those with mostly standing type of work were having lesser insufficiencies as compared to others. Positive participants included students (20\%), office workers $(23 \%)$, Labours (7\%) and housewives (30\%). However, $5 \%$ of the students, $10 \%$ of the office workers and $13 \%$ of the house wives were reported to have severe difficulties with their muscular functions.

Among 150 subjects, 119 individuals are those who had a complaint of neuromuscular indications includes body pain, joint's pain, back pain, numbness and general muscle weakness out of the 48 individuals reported to have musculoskeletal difficulties includes difficulty in walking, running, holding and strength.

Indications and difficulties regarding neuromuscular disorders have closed relation with the age, gender, consanguineous marriages, origin and types of work. The risk of such indications increases with the increasing age and kind of work. In this study, history of neuromuscular disorder was mostly found in the subjects who were having consanguineous marriages among their parents as well as indications and difficulties of the neuromuscular disorder are commonly observed in the subjects who had family and diagnosed history of a neuromuscular disorder. This study showed that body pain has the highest ranking (68\%) and back pain has the second-highest ranking $(52 \%)$ that have been reported in our subjects.

We had reported the highest compliant of neuromuscular indications from the middle-aged group than the young and old-aged group and highest complaint of neuromuscular difficulties in the old-aged group than young -aged and middle-aged group. In the middle-aged people we had found work-related occupational disorders. Occupational disorders cause pain in upper extremities, which might be result from the combination of physical and psychological factors. Excessive work load with repetitive working cause tissues damages that lead to pain and illness. Certain other factors that cause severity in pain include anxiety, depression, disabilities and somatic sensation. In old-aged people difficulties were mostly seen with indications because advancing age reduces the performance of muscles. According to the theory of Burke, Tuttle, Thompson, Janney and Webber that between age of 25-79 the maximum grip of normal muscle will reduces to half because of muscle wasting especially proximal limb and hand muscles which indicates that number of motor neuron will reduces.

The kind of work, type of work and duration of work has great impact on structure and functions of muscles. In this study mostly neuromuscular complaints were recorded from house wives and office workers with low back pain and neck pain. Low back pain is commonly observed in the professions such as banker, clerks, accountant, industrial workers and computer and visual screen users because of prolonged sitting on chair as their part of job. Prolonged sitting with repetitive work and immobile posture without changing position while using computer and visual screen become more common now a days in our society, which cause affect y to spine structure (cervical and lumber region) due to improper angle posture which result in low back pain because during prolonged sitting our spine suffers from stress. Thus, musculoskeletal complaints were commonly observed in office workers (Khan et al,. 2005).

This study shows the high prevalence of body pain and low back in students, office workers, house wives and labours that have mostly sitting kind of work. In students, prolonged sitting had seen during studies, reading, labs works and making assignment is very common. Several researches showed that due to inadequate knowledge regarding poor posture among students result in postural problems [8]. About 70\% student is affected by cervicogenic pain. The prevalence of cervicogenic pain is more common in females as compare to males. Some risk factors that contribute to cervicogenic pain are soft tissue related disorder and structural changes in cervical inter-vertebral disc, facet joint, atlanto-axial and atlanto-occipital joint due to prolonged sitting in particular position. Students mostly sit during studies in such a position that they bend their head which increase the load on cervical muscles in order to maintain the balance of head against gravity result in cervicogenic pain and musculoskeletal disorder [9]. In office workers, due to six to eight hours of working, they mostly reported to have low back pain with hamstring muscles extensibility because of prolonged sitting without any break. Hamstring muscles are the muscles of thigh which responsible for the extensor of hip and flexor of toe. In office worker due to prolong as their job requirement, hamstring muscles lose their flexibility because of shortening and tightness which lead to muscle atrophy [10].

Our studies demonstrated that the high prevalence of body pain including neck ache and back aches among visual screen users more than 5 hours. Visual screen has become the basic life need from past few years' example television which has been used during leisure time and computer which has been used by students and office workers which find to have an association with neck pain. Neck pain is a common problem reported by visual screen users because of continuous use of computers in their daily life with bend neck in forward direction and extend sitting in specific posture cause stretch in neck muscles that result in muscle imbalance. Neck pain is experienced at the base skull, shoulder and upper parts of back. Symptom of neck pain includes continuous ache in neck, shoulder, arm and feeling of discomfort in the region of neck and shoulder. The risk factors that cause pain include stress, improper posture and daily four to five hours of computer use [11]. 


\section{Biosight 2020; 01(02): 16-23}

This study shows the low intake of water habit among individuals that reported to have neuromuscular indications and associated difficulties. Individual should have to take an adequate amount of water during summer or winter seasons to urinate. It has been seen that low degree of dehydration also cause decrease working ability and prolonged dehydration also causes weakness, fatigue, nausea, vomiting and headaches during hot climate. Dehydration might be result from heat exhaustion, alcohol and beverages include tea, coffee and soft drink.

We also observed individuals those are addicted certain addiction including soft drink, betel, chewing leaves, smoking and gutka were reported to have neuromuscular indication and difficulties. Areca nuts, betel, chewing leaves, tobacco, alcohol and caffeine addictions is most commonly seen in Pakistani population that cause oral squamous cell carcinoma (cancer) among individuals. These addictions are also the risk factors of certain diseases such as leukoplakia, circulatory, pulmonary and metabolic disturbance that effect osmotic pressure, neurotransmission, intestinal movement and physiology of gut, lungs and pancreases [12].

In this study we also observed high prevalence of using self medication in female gender including house wives and students by means of treatment against certain pathological condition. Self medication is defined as use of any medicine or pharmaceutical product without any doctor prescription. In many under developing countries including Pakistan culture of self-medication is very common. People used medicine with non-prescription in certain disease condition. Medicine that are easily available at pharmacies includes pain killers, analgesics, vitamins, cough-suppressant and anti-allergic. High self medication ratio among Pakistani population is might be because of easily available medicine at pharmacies, expensive health care services, lack of knowledge about adverse side effects of these drugs [13]

\section{Conclusion:}

Neuromuscular indication and difficulties are increasing rapidly without being diagnosed properly. Our life style and daily routine is playing an important role, especially lack of exercise, lesser water intake and more exposure to screens. Back pain is the major complain recorded through this data which might because of prolonged inappropriate posture.

\section{Ethics Approval and Consent To Participate}

Not applicable.

\section{Human and Animal Rights}

Not applicable.

\section{Consent for Publication}

Not applicable.

\section{Availability of Data and Materials \\ None}

Funding

None

\section{Conflict of Interest}

The authors declare no conflict of interest, financial or otherwise.

\section{Acknowledgements}

None

\section{References}

1. Turakhia P, Barian B and Jeffey B. Patient with neuromuscular disorder. Med Clin North Am 2013; 97(6): 1015-32.

2. Laing NG. Genetics of neuromuscular disorder. Critic Rev Clin Lab Sci 2012; 49(2): 33-48.

3. Buskila D, Lily N, and Joseph P. Genetic factors in neuromuscular pain. CNS Spectrum 2005; 10(4): 281-4.

4. Katiriji B, Henry J, Kaminski and Robert L. Physical management of patient with neuromuscular diseases. Neuromuscular Disease in Clinical Practice, 2nd edition. Springer London 2013: 327 8.

5. Campbell MJ, Mccomas AJ and Petito F. Physiological changes in aging muscles. J Neurol Neurosurg Psychiatry 1973; 36: 174-82. 


\section{Biosight 2020; 01(02): 16-23}

6. Cory PC. Consanguinity and prevalence patterns of inherited diseases in the UK Pakistani Community. Human Hereditary 2017; 77: 207-216.

7. Khan MA and Ghouri AM. Environmental pollution: Its effects on life and its remedies. J Arts Sci Commerce 2011; 2(2): 276-85.

8. Farooqui SI, Amna AK, Maira R, Nazish R and Ali F. Analyzing spinal curves for prolonged sitting in university going student. Eur Int J Sci Technol 2015; 4(7): 58-65.

9. Navaid-us-Saba, Amna AK, Sumaira IF and Zara O. The association of sitting posture and cervicogengic pain among the students of physical therapy. Pakistan J Rehabilitation 2012; 1(1): 44-9.

10. Waqas MS, Mazher A, Saba H and Sajid M. Frequency of Reduced Hamstring Flexibility in Prolonged Sitting (6-8 hours) among Office Workers. Journal Riphah College of Rehabilitation Sci 2016; 4(2): 36-9.

11. Sabeen F, Muhammad SB, Imtiaz S and Sarah E. Prevalence of Neck Pain in Computer Users. Annals of King Edward University Lahore, Pakistan, 2013. 19(2): 137-143.

12. Khan MA, Saima S and Syed MS and Hameed A. Prevalence of Oral Squamous Cell Carcinoma in Relation to Different Chewing Habit in Karachi, Pakistan. Pak J Biochem Mol Bio 2012; 45(2): 59-63.

13. Khan H, Safirah M, Alamgeer GA, Asif M and Muhammad R. Determinants of Increasing Trend of Self Medication in a Pakistani Community. Tropical J Pharm Researches 2014; 13(3): 437-43. 